\title{
Impaired DNA double-strand break repair contributes to the age-associated rise of genomic instability in humans
}

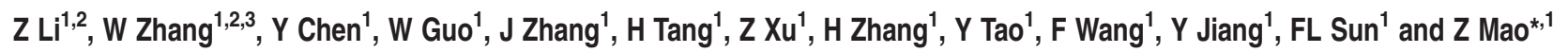

Failing to repair DNA double-strand breaks by either nonhomologous end joining (NHEJ) or homologous recombination (HR) poses a threat to genome integrity, and may have roles in the onset of aging and age-related diseases. Recent work indicates an age-related decrease of NHEJ efficiency in mouse models, but whether NHEJ and HR change with age in humans and the underlying mechanisms of such a change remain uncharacterized. Here, using 50 eyelid fibroblast cell lines isolated from healthy donors at the age of 16-75 years, we demonstrate that the efficiency and fidelity of NHEJ, and the efficiency of HR decline with age, leading to increased IR sensitivity in cells isolated from old donors. Mechanistic analysis suggests that decreased expression of XRCC4, Lig4 and Lig3 drives the observed, age-associated decline of NHEJ efficiency and fidelity. Restoration of XRCC4 and Lig4 significantly promotes the fidelity and efficiency of NHEJ in aged fibroblasts. In contrast, essential HR-related factors, such as Rad51, do not change in expression level with age, but Rad51 exhibits a slow kinetics of recruitment to DNA damage sites in aged fibroblasts. Further rescue experiments indicate that restoration of XRCC4 and Lig4 may suppress the onset of stress-induced premature cellular senescence, suggesting that improving NHEJ efficiency and fidelity by targeting the NHEJ pathway holds great potential to delay aging and mitigate aging-related pathologies.

Cell Death and Differentiation (2016) 23, 1765-1777; doi:10.1038/cdd.2016.65; published online 8 July 2016

Aging in mammals is a complex biological process, characterized by several major hallmarks., ${ }^{1,2}$ Of all the features associated with aging, a gradual destabilization of genome integrity is perhaps the most fundamental as increased genomic instability may lead to other age-associated phenotypes such as cellular senescence and stem cell exhaustion. Indeed, for the past several decades, numerous studies have indicated that DNA mutations and chromosomal rearrangements gradually accumulate with age.$^{3-6}$ Of all types of DNA lesions, which may contribute to the gradual loss of genetic information during aging, DNA double-strand breaks (DSBs) are the most hazardous to cells as unrepaired or inappropriately repaired DSBs can cause insertions, deletions and chromosomal rearrangements. Using different analysis approaches, several studies have demonstrated that aging is often associated with the accumulation of DNA DSBs in various organs and tissues in mammals such as mice and humans. ${ }^{7-11}$ Moreover, a recent study provides direct evidence that an induction of DNA DSBs in genomes causes aging in mouse livers. ${ }^{12}$ However, why DNA DSBs accumulate with age remains an open question. A number of studies indicate that it may be a consequence of a progressing imbalance between DNA damage and the efficiency of the molecular machinery that catalyzes DNA repair. ${ }^{7,9,11}$

Two major pathways, nonhomologous end joining (NHEJ) and homologous recombination (HR) evolved to repair DNA DSBs. NHEJ is further divided into two sub-pathways: the classical NHEJ pathway (c-NHEJ)—in which the major participating factors include DNA-PKcs, Ku70/Ku80 heterodimers, Artemis, XRCC4, XLF and DNA Lig4-and the alternative NHEJ pathway (alt-NHEJ), comprised of the repair factors, PARP1 and DNA Lig3. ${ }^{13,14}$ Both NHEJ pathways are mutagenic, with C-NHEJ resulting in deletions and insertions, whereas alt-NHEJ leading to the loss of genetic information between micro-homologies on chromosomes. In contrast to NHEJ, which is error-prone and occurs throughout the whole cell cycle, the HR pathway is theoretically precise and only takes place in S/G2 stages when sister chromatids are available. ${ }^{15}$ The core components taking part in this process are the MRN complex, BRCA1, CtIP, the recombinase Rad51, Rad51 paralogs, WRN and several others. ${ }^{16}$ Knocking out NHEJ or HR factors, such as DNA-PKcs, Ku70, Ku80, Artemis or WRN leads to an accelerated aging phenotype in mice, ${ }^{4,17}$ strongly suggesting that the two pathways have essential roles

\footnotetext{
${ }^{1}$ Clinical and Translational Research Center of Shanghai First Maternity \& Infant Hospital, Shanghai Key Laboratory of Signaling and Disease Research, School of Life Sciences and Technology, Tongji University, Shanghai 200092, China

${ }^{*}$ Corresponding author: Z Mao, Clinical and Translational Research Center of Shanghai First Maternity \& Infant Hospital, Shanghai Key Laboratory of Signaling and Disease Research, School of Life Sciences and Technology, Tongji University, 1239 Siping Road, Shanghai 200092, China. Tel: +86 21 65978166; Fax: +86 21 65981041; E-mail: zhiyong_mao@tongji.edu.cn

${ }^{2}$ These authors contributed equally to this work.

${ }^{3}$ Current address: Department of Plastic Surgery, Changzheng Hospital, \#415 Fengyang Road, Shanghai 200003, China.

Abbreviations: DSB, double-strand break; NHEJ, nonhomologous end joining; HR, homologous recombination; alt-NHEJ, alternative NHEJ; c-NHEJ, classical NHEJ; SSA, single-strand annealing; SIPS, stress-induced premature senescence; GFP, green fluorescent protein; Ad2, adenoviral exon 2; SD, splicing donor; SA, splicing acceptor; IR, ionizing radiation; PD, population doubling

Received 26.10.15; revised 09.5.16; accepted 10.6.16; Edited by M Oren; published online 08.7.2016
} 
Table 1 The age distribution of donors

\begin{tabular}{lcc}
\hline Age group & Number of donors & Percentage of donors (\%) \\
\hline$<20$ & 6 & 12 \\
$<30-\geqslant 20$ & 12 & 24 \\
$<40-\geqslant 30$ & 5 & 10 \\
$<50-\geqslant 40$ & 8 & 16 \\
$<60-\geqslant 50$ & 10 & 20 \\
$\geqslant 60$ & 9 & 18
\end{tabular}

Volunteers who donated the eyelid samples after plastic surgeries are all healthy females. Over 100 eyelid tissues were collected and fibroblast lines derived from them were established. Fifty cell lines were then randomly selected for this study

in aging. Not limited to mice, several progeria syndromes in humans including Werner syndrome and A-T syndrome are all linked to the reduced capability of repairing DNA DSBs. ${ }^{17}$

Here, we successfully isolated 50 eyelid fibroblast cell lines from female donors between the age of 16 and 75 years. With these cell lines, using easy-to-score fluorescence-based assays for quantifying NHEJ and HR, we found that both the efficiency and fidelity of NHEJ, as well as the efficiency of HR significantly decline with age. Further mechanistic studies suggest that important NHEJ factors, XRCC4, DNA Lig4 and DNA Lig3, exhibit an age-related change in protein expression level. Restoration of XRCC4 and DNA Lig4 rescues the decline in NHEJ efficiency and fidelity. In contrast, the sharp decline of HR with age is caused not by changes in cell cycle or major HR protein expression, but possibly by the impaired recruitment of Rad51 to DNA damage sites. As NHEJ, rather than HR, is a dominant pathway during DNA DSB repair, we propose that targeting the NHEJ pathway, particularly XRCC4 and DNA Lig4, is a potential way to delay aging and mitigate age-associated pathologies by promoting genomic stability.

\section{Results}

Efficiency of NHEJ and HR declines with age. To test the influence of aging on DNA DSB repair in humans, we first isolated 50 fibroblast cell lines from the eyelid tissue of female donors at the age of 16-75 years according to our wellestablished protocol (Table 1). ${ }^{18,19}$ The isolated fibroblasts were cultured in proper conditions to minimize potentially harmful stress to primary cells. In addition, to rule out the effect of replicative cellular senescence on DNA repair, all the following analysis in this report was performed at a population doubling (PD) number of 16-19 in all cell lines (Supplementary Table S1).

The GFP-based reporter cassettes for measuring NHEJ and $\mathrm{HR}$ efficiency have been well recorded in previous research (Figure 1a). ${ }^{15,20,21}$ The NHEJ reporter contains a GFP gene with a rat Pem1 intron (GFP-Pem1) and an adenoviral (AD) exon flanked by two non-palindromic I-Scel recognition sites in an opposite orientation. The intact NHEJ reporter is GFP negative as the $A D$ exon is spliced into the GFP exon at mRNA level, killing the GFP activity at protein level. The HR construct consists of two copies of defective GFP-Pem1. The first exon of GFP in the first copy is killed by a 22-nucleotide deletion and two inverted-oriented I-Scel recognition sites, whereas the second copy of GFP-Pem1 is killed by a lack of ATG start codon and second exon of GFP. Therefore, the intact HR reporter is also GFP negative.

To analyze the efficiency of NHEJ or HR in a quantitative way, we generated NHEJ or HR reporters with DNA DSBs by linearizing them with in vitro I-Scel digestion, and transfected them to the 50 cell lines together with a DsRed plasmid for normalizing the transfection efficiency. At $72 \mathrm{~h}$ post transfection, cells were collected for the FACS analysis. The ratio of GFP+ cell number versus DsRed+ cell number was used as the measure of NHEJ or HR efficiency. Using this extrachromosomal assay, we systematically tested the NHEJ and HR efficiency in the 50 cell lines. The results reveal an extremely significant, age-related decline of both NHEJ and HR efficiency (NHEJ, $r^{2}=0.24, P<0.0003$; $\mathrm{HR}, r^{2}=0.52$, $P<0.00000001$ ) (Figures $1 \mathrm{~b}$ and $\mathrm{c}$ ), suggesting that changes in NHEJ and HR efficiency during aging might participate in the onset of aging. Moreover, we observed an extremely significant correlation between NHEJ and HR efficiency $\left(r^{2}=0.47, P=0.00000003\right)$ (Supplementary Figure S1), suggesting that NHEJ and HR may share common regulatory mechanisms, possibly in the initial stages of the DNA damage response pathway.

Accuracy of NHEJ declines with age. Intriguingly, although NHEJ, instead of HR, is the dominant pathway in mammals, and knocking out NHEJ factors rather than HR factors leads to premature aging in mice, HR efficiency exhibits a sharper decline than NHEJ with age, implying that other than the efficiency of NHEJ, other aspects of NHEJ, such as NHEJ fidelity, might also change with age. To test this hypothesis, we randomly picked 14 young cell lines (16-29 years) and 14 aged cell lines (50-75 years) from the 50 cell lines (Supplementary Figure S2). Using a similar approach to the analysis of the NHEJ efficiency, we transfected the linearized NHEJ reporter to the 28 cell lines and then collected cells for DNA extraction. After extraction, the DNA, including the repaired NHEJ plasmids, was transformed to E.coli, the repaired products were isolated and digested with $\mathrm{Xbal}$ restriction enzyme. If repaired in a relatively precise way, the digested plasmids should have two bands with a size of $\sim 720 \mathrm{bp}$ and $\sim 6.6 \mathrm{~kb}$ (Figure 2a). If repaired in an inaccurate way, $X$ bal digestion would result in either two bands with a size of much smaller than $720 \mathrm{bp}$ and $\sim 6.6 \mathrm{~kb}$, or with only one band, or with uncut construct, leading to a supercoil on the agarose gel. Using this assay, our analysis indicates that NHEJ is more precise in young cells than that in aged cells (Figure 2a). To further examine the fidelity of NHEJ in a more quantitative way, we sequenced the junctions of the rescued plasmids and compared the fidelity between the two groups. Although the process of NHEJ is often associated with deletions or insertions, ${ }^{4,21,22,23}$ the chance of acquiring insertions in eyelids fibroblasts is extremely slim (0-15\%) (Supplementary Table S2), so it is not feasible to analyze the change of insertion sizes with age in these cell lines. We therefore systematically compared the sizes of deletions at the junction areas. For each cell line, at least 20 rescued plasmids with deletions were analyzed as previously reported. ${ }^{21}$ The average deletion size of the 14 young cell lines is $463 \mathrm{bp}$, whereas the 14 aged cell lines have an average deletion size of $663 \mathrm{bp}$. We grouped the clones into 
a NHEJ

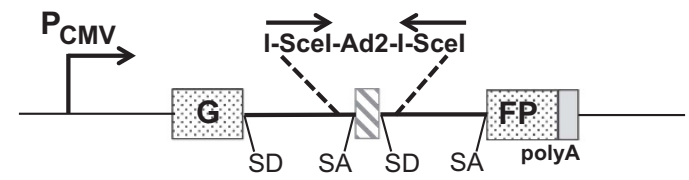

HR

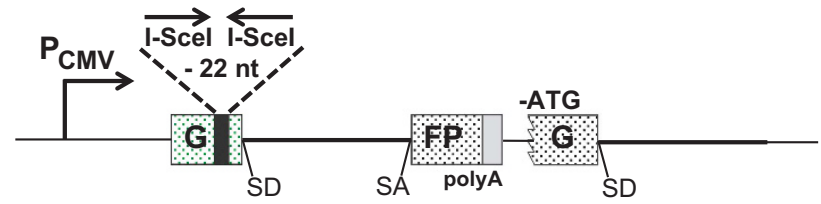

b
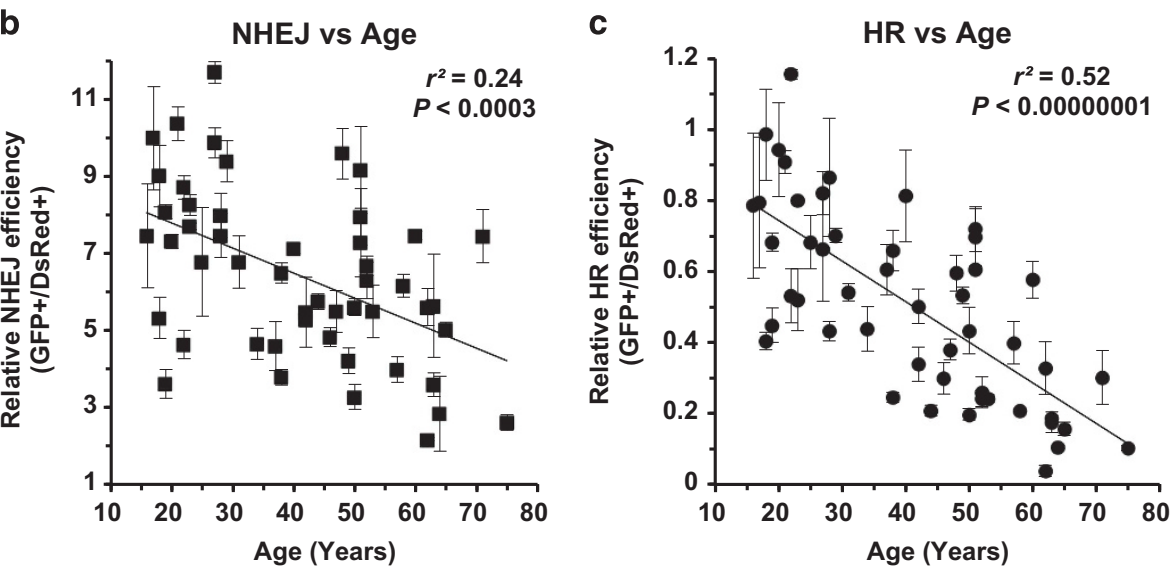

Figure 1 The efficiency of DNA DSB repair by both NHEJ and HR declines significantly with age. (a) Reporter substrates for analysis of NHEJ and HR efficiency. Both reporter cassettes were constructed on the basis of EGFP. The NHEJ construct is composed of one copy of EGFP with a Pem1 intron and an Ad2 exon, which inactivates EGFP expression at the transcriptional level. DNA DSBs can be induced by the I-Scel restriction enzyme, which cuts the two I-Scel recognition sites in an opposite orientation flanking the Ad2 exon. Successful NHEJ restores the EGFP expression, which may be scored on FACS. The HR construct contains two copies of an inactivated EGFP-Pem1. In the first copy, the first exon of GFP was engineered with a 22-nt deletion and two inverted I-Scel recognition sites. The second copy lacks the ATG start codon and the second exon of GFP. After a DNA DSB is induced on the HR reporter, only gene conversion, which predominates HR pathway, restores EGFP activity. (b) A significantly negative correlation between NHEJ efficiency and age.

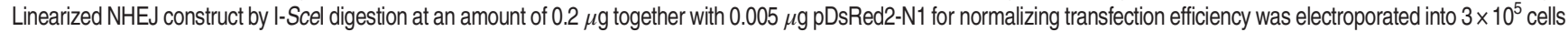
at exponentially growth stage. Cells were collected for the analysis on FACS at $72 \mathrm{~h}$ post transfection. Error bars, S.D. (c) A significantly negative correlation between HR efficiency

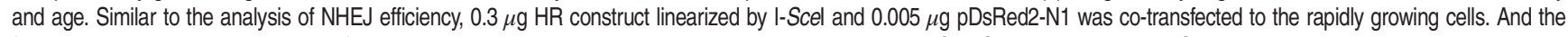
following analysis is identical to that of NHEJ. All the experiments were repeated 3-12 times. Error bars, S.D. SA, splicing acceptor; SD, splicing donor

three classes as shown in Figure $2 b$, and we found that frequency of acquiring large deletions (>500 bp) in the group of young cells is significantly lower, whereas the frequency of acquiring small deletions ( $<100 \mathrm{bp}$ ) is higher than that in the group of old cells (Figure 2c), suggesting that aged eyelid fibroblasts tend to generate larger deletions during DNA DSB repair by NHEJ.

Genomic instability rises with age in human eyelid fibroblasts. To understand the consequences of impaired NHEJ and HR in the context of genome integrity, we further examined the formation of $\mathrm{yH} 2 \mathrm{Ax}$ foci, which is often characterized as the repair center of DNA DSBs and therefore is used widely as the marker of DNA DSBs, at different time points post infrared (IR) treatment (X-ray, $8 \mathrm{~Gy}$ ) in the young and old group of cell lines. We found that the number of $\gamma \mathrm{H} 2 \mathrm{Ax}$ foci formed at $2 \mathrm{~h}$ post IR does not change in the group of young cells compared to that in old group (Figures $3 a$ and b; Supplementary Figure S3), suggesting that age probably does not have a role in the detection of DNA DSBs. Intriguingly, probably as a result of declining capacity of DNA DSB repair, we observed a significant increase of $\gamma \mathrm{H} 2 \mathrm{Ax}$ foci in aged cell lines in comparison to that in young cell lines (Figures $3 b$ and $c$ ), indicating that more DNA damages remain unrepaired in the old group.

However, possibly due to the age-related changes of chromatin structure, which influences the foci formation of $\gamma \mathrm{H} 2 \mathrm{Ax},{ }^{24}$ under normal growth condition with no IR treatment to cells, we did not observe significant difference of $\gamma \mathrm{H} 2 \mathrm{Ax}$ foci number between the two groups (Figure $3 b$ ). We therefore tested the genome integrity using neutral comet assay, which only measures the relative amount of DNA DSBs per cell. In addition, as the high salt and detergent destroys the chromatin structure on the DNA, this assay is not influenced by chromatin structures. Indeed, we found that the average tail moment of 14 young cell lines is 3.23 , whereas that of the 14 old cell lines is $\sim$ twofold higher (average tail moment $=6.33$ ). Statistical analysis indicates that the tail moment in the group of aged cells is significantly increased compared to that in the young cell lines (Figures $3 d$ and e). We then further examined whether there is a correlation between the tail moment and DNA repair efficiency. We found both NHEJ and HR efficiency negatively correlates with tail moments (Figures $3 f$ and $g$ ), 

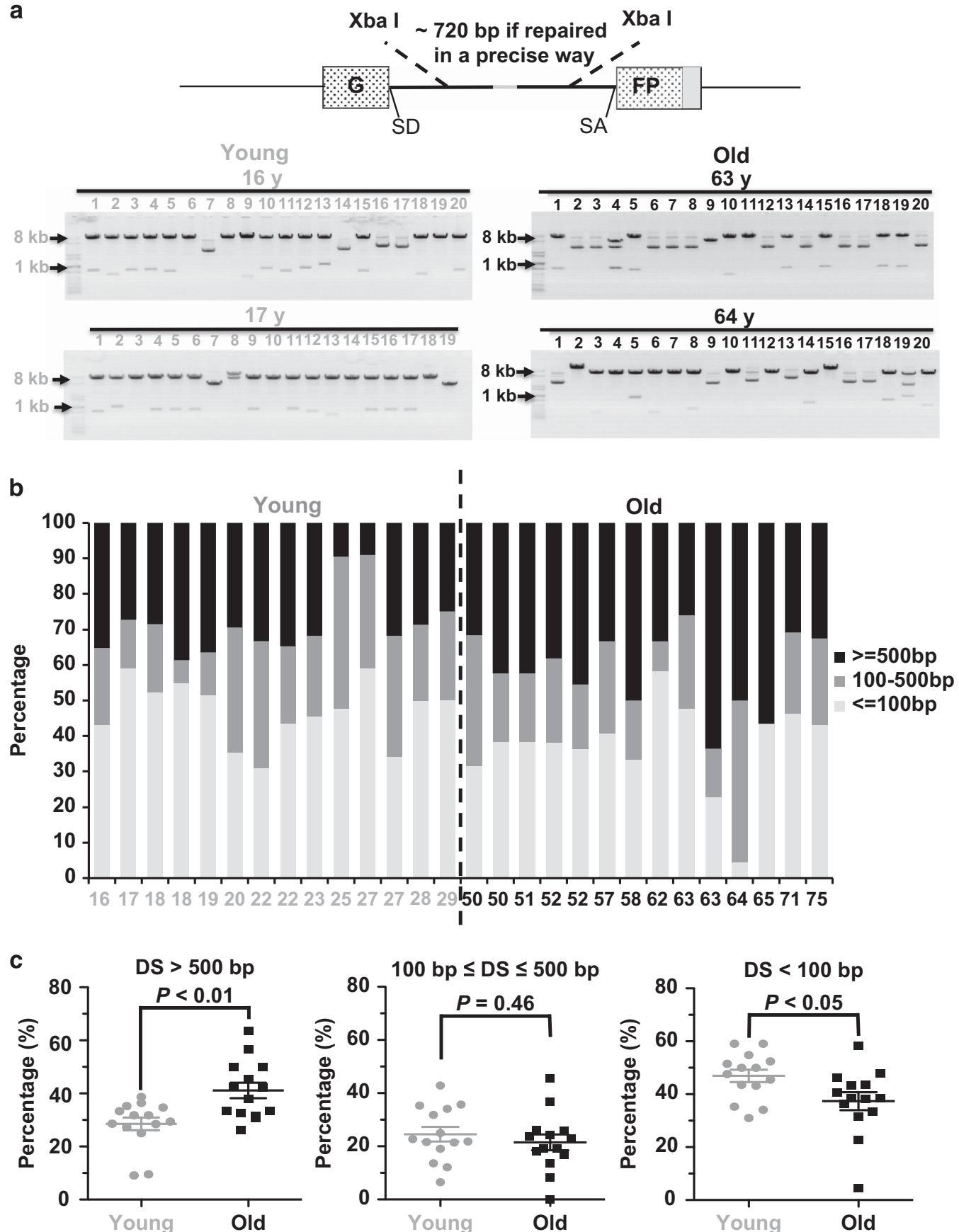

Figure 2 NHEJ becomes more error-prone during aging. (a) A depiction of NHEJ fidelity analysis. At $72 \mathrm{~h}$ post transfection of $0.6 \mu \mathrm{g}$ of linearized NHEJ construct into $10^{6}$ cells, cells were collected for plasmid rescue. The rescued NHEJ plasmids were isolated and digested with Xbal restriction enzyme. Two Xbal recognition sites sit downstream and upstream of break sites, so the size of DNA bands after Xbal digestion would reflect the relative fidelity of NHEJ. Twenty rescued plasmids were isolated and digested with Xbal. Two representative gel pictures from two cell lines from each group are shown. (b) The frequency of acquiring different sizes of deletions in the 28 cell lines. For each cell line, at least 20 clones with deletions were sequenced at the junction areas. (c) The old group of cells are more likely to have large deletions but less prone to have small deletions than the young group of cells. The Mann-Whitney $U$ (MWU) test indicates that the differences are significant. DS, deletion sizes

confirming that a declined DNA repair efficiency is probably responsible for the rise of genomic instability. Intriguingly, the correlation between NHEJ fidelity and tail moment is not significant (Supplementary Figure S4), possibly because tail moment can only reflect relative amounts of DNA DSBs rather than the impairment of genetic information.
Radiosensitivity rises with age in human eyelid fibroblasts. To further examine the functional consequences of the age-associated decline of DNA DSB repair, we compared the IR sensitivity of the two groups of cells by performing a clonogenic assay. We calculated the $\mathrm{LD}_{50}$ and $\mathrm{LD}_{75}$ after $\mathrm{X}$-ray treatment for the young and old cells (Figures $4 a$ and $b$ ). 
a

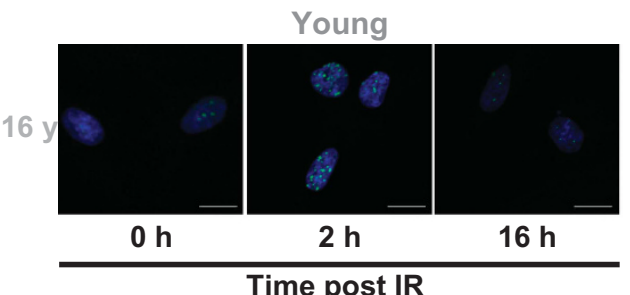

b

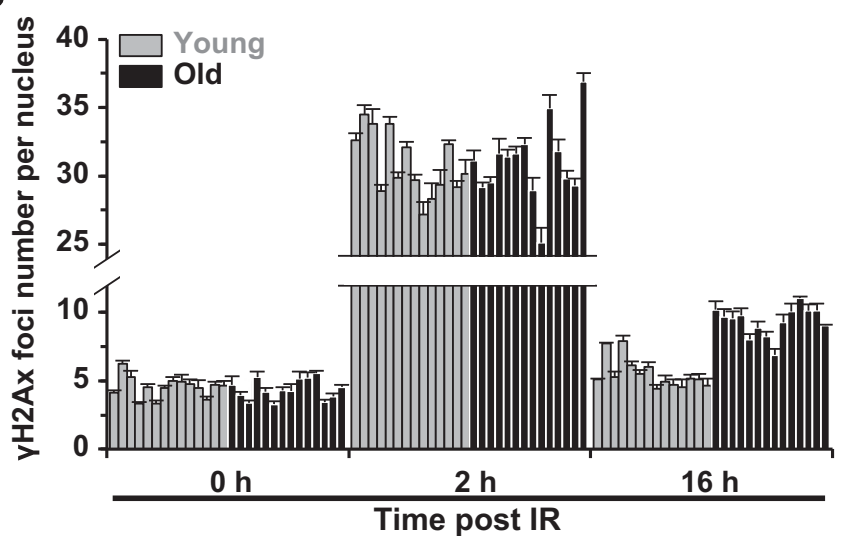

d

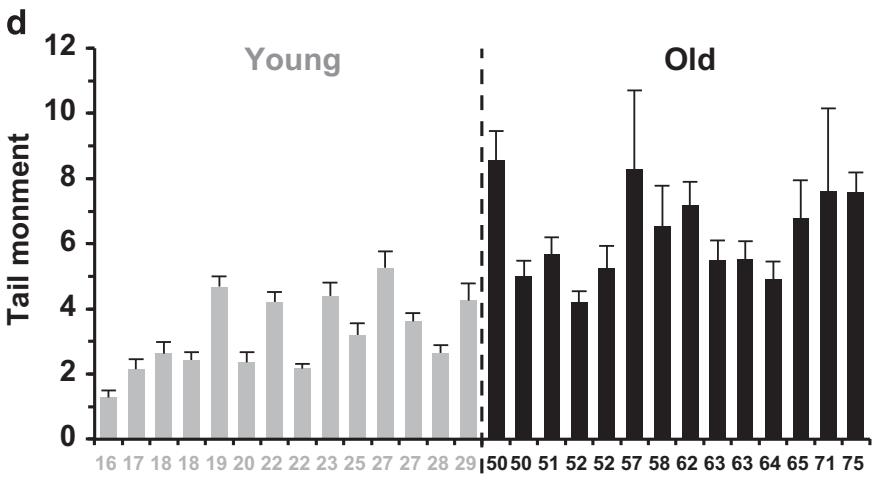

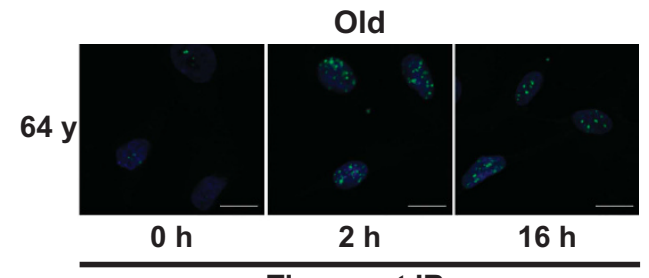

Time post IR

c

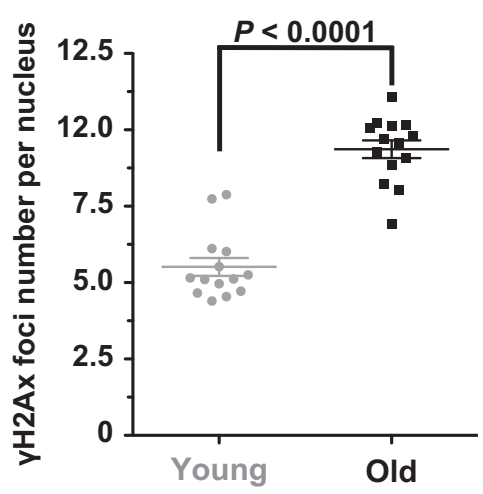

e

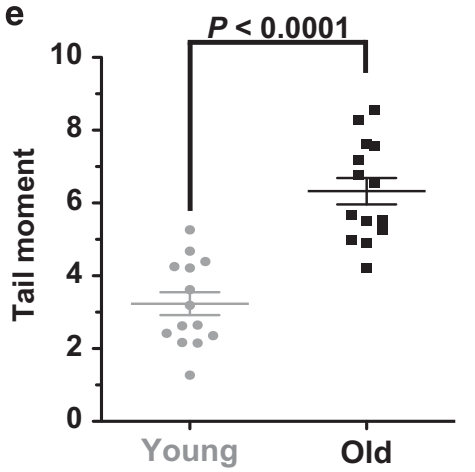

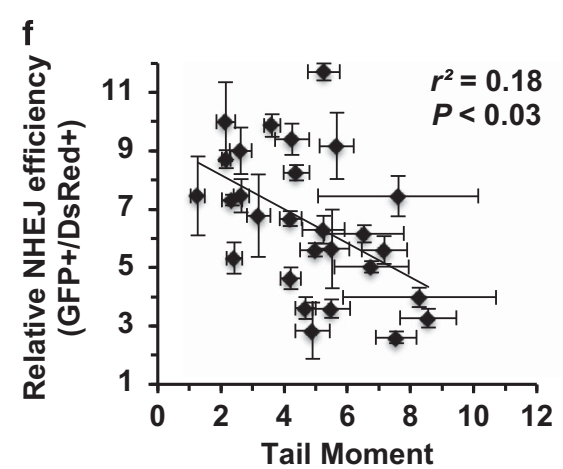

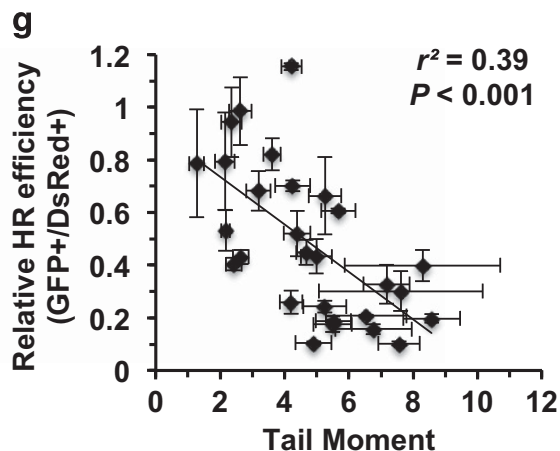

Figure 3 Age-related decline of DNA DSB repair efficiency strongly correlates with genomic instability. (a) Representative pictures of $\gamma \mathrm{H} 2 \mathrm{Ax}$ foci at different time points post $\mathrm{IR}$ (X-ray, $8 \mathrm{~Gy}$ ) in one young and old cell line. Scale bars, $10 \mu \mathrm{m}$. (b) Quantification of $\gamma \mathrm{H} 2 \mathrm{Ax}$ foci numbers at the three time points in the 28 cell lines. At least 50 cells were counted for each time points. Error bars, S.E.M. (c) Statistical analysis (MWU test) reveals that the old group of cells have a significantly higher number of $\gamma \mathrm{H} 2 \mathrm{Ax}$ foci at $16 \mathrm{~h}$ post IR than the young group of cells. (d) Comparison of genomic instability measured by neutral comet assay between the two groups. The 28 cell lines were analyzed at PD $16-19$ using a kit from Trevigen. For each cell line, the tail moments of at least 50 cells were quantified using the Cometscore software (Sumerduck, VA, USA). Error bars, S.E.M. (e) MWU test reveals that the old group of cells have significantly higher tail moments than the young group. (f) There is a significant negative correlation between NHEJ efficiency and tail moment. Error bars of tail moment, S.E.M. Error bars of NHEJ efficiency, S.D. (g) There is a significant negative correlation between HR efficiency and tail moment. Error bars of tail moment, S.E.M. Error bars of HR efficiency, S.D. 

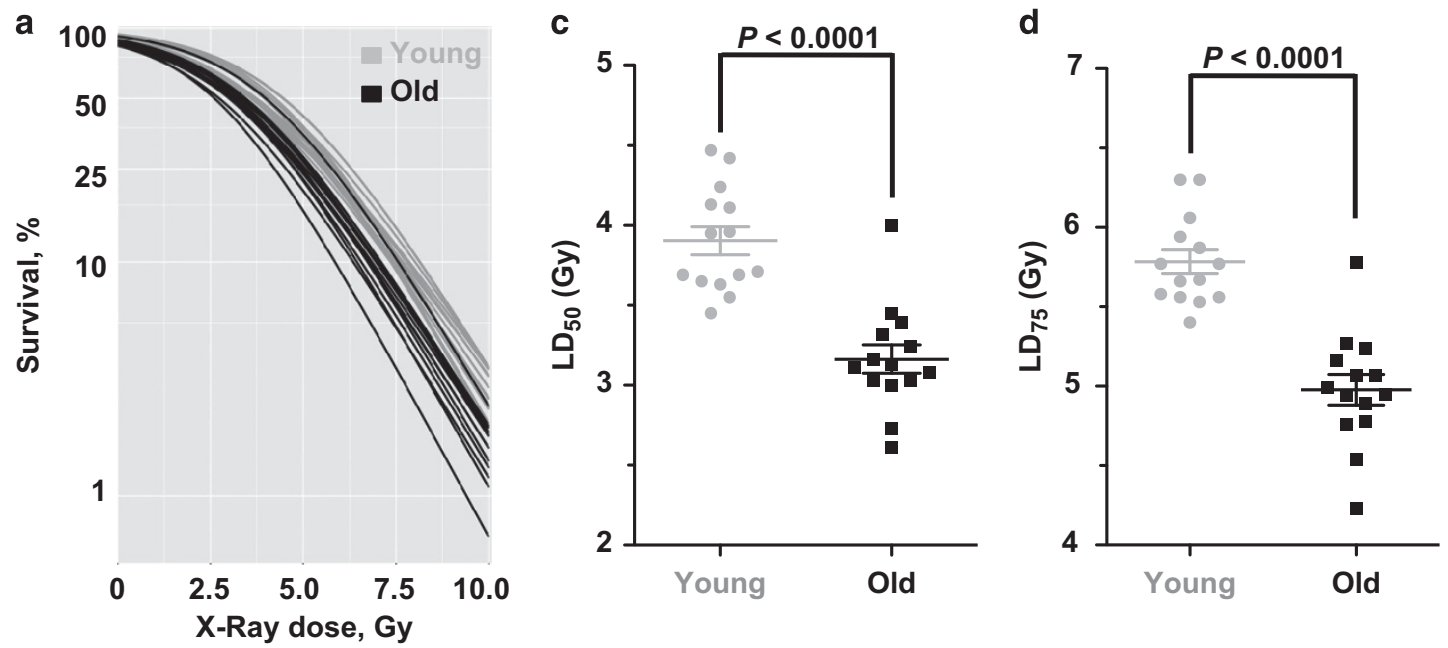

b

\begin{tabular}{|c|c|c|}
\hline Age & LD $50(95 \% \mathrm{Cl})$ & $\mathrm{LD}_{75}(95 \% \mathrm{Cl})$ \\
\hline 16 & $4.47(3.88-5.06)$ & $6.30(5.52-7.08)$ \\
\hline 17 & $3.69(3.02-4.37)$ & $5.77(4.90-6.65)$ \\
\hline $18-1$ & $4.24(3.56-4.93)$ & $5.94(5.06-6.82)$ \\
\hline $18-2$ & $4.13(3.60-4.66)$ & $5.87(5.18-6.55)$ \\
\hline 19 & $4.42(3.86-4.97)$ & $6.23(5.50-6.97)$ \\
\hline 20 & $3.45(2.83-4.08)$ & $5.40(4.62-6.18)$ \\
\hline $22-1$ & $3.63(3.02-4.25)$ & $5.56(4.77-6.34)$ \\
\hline $22-2$ & $3.71(3.12-4.30)$ & $5.56(4.81-6.31)$ \\
\hline 23 & $4.11(3.49-4.74)$ & $6.06(5.24-6.89)$ \\
\hline 25 & $3.75(3.10-4.39)$ & $5.77(4.93-6.60)$ \\
\hline $27-1$ & $3.96(3.44-4.48)$ & $5.66(5.00-6.32)$ \\
\hline $27-2$ & $3.55(2.92-4.19)$ & $5.58(4.77-6.39)$ \\
\hline 28 & $3.95(3.44-4.47)$ & $5.67(5.02-6.32)$ \\
\hline 29 & $3.65(3.01-4.29)$ & $5.53(4.72-6.33)$ \\
\hline $50-1$ & $3.16(2.50-3.82)$ & $5.03(4.23-5.84)$ \\
\hline $50-2$ & $3.32(2.71-3.94)$ & $5.16(4.41-5.92)$ \\
\hline 51 & $3.45(2.83-4.08)$ & $5.27(4.49-6.04)$ \\
\hline $52-1$ & $3.24(2.62-3.87)$ & $4.99(4.23-5.75)$ \\
\hline $52-2$ & $3.13(2.41-3.85)$ & $5.07(4.19-5.94)$ \\
\hline 57 & $2.61(1.91-3.31)$ & $4.23(3.42-5.04)$ \\
\hline 58 & $2.73(2.02-3.45)$ & $4.54(3.70-5.39)$ \\
\hline 62 & $3.03(2.32-3.74)$ & $4.94(4.08-5.80)$ \\
\hline $63-1$ & $3.00(2.36-3.65)$ & $4.78(4.01-5.56)$ \\
\hline $63-2$ & $4.00(3.49-4.50)$ & $5.78(5.13-6.42)$ \\
\hline 64 & $3.39(2.77-4.01)$ & $5.24(4.47-6.01)$ \\
\hline 65 & $3.03(2.36-3.70)$ & $4.89(4.08-5.70)$ \\
\hline 71 & $3.08(2.48-3.68)$ & 4.76 (4.04-5.49) \\
\hline 75 & $3.11(2.48-3.75)$ & $4.95(4.18-5.72)$ \\
\hline
\end{tabular}
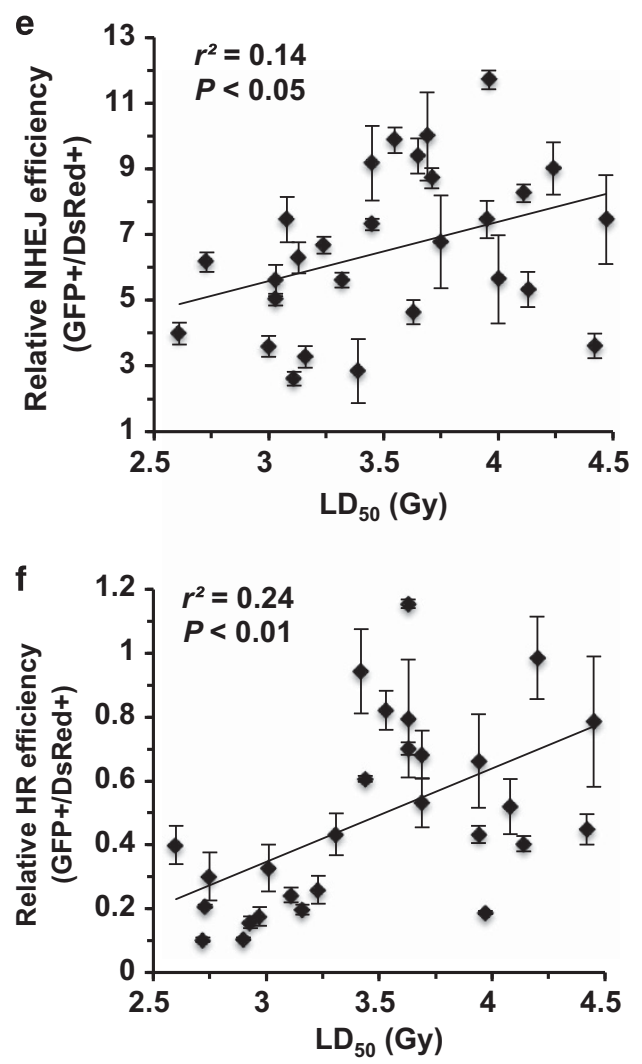

Figure 4 Age-related decline of DNA DSB repair strongly correlates with radiosensitivity. (a) Young and old cells were irradiated with increasing doses of X-ray. Survival was calculated as the relative plating efficiencies of irradiated versus control, unirradiated cells. Logistic regression was performed separately on the 28 samples to estimate the cell's sensitivity to IR. The experiment was performed in triplicate for each sample. (b) Sensitivity of eyelid fibroblasts to X-ray (Gy). The lethal dose that kills 50 and $75 \%$ of the population of a test sample's cells were calculated by the logistic regression. (c,d) The MWU test indicates that the young group of cells has a statistically significant higher $\mathrm{LD}_{50}$ and $L_{75}$ than that of the old cells. (e) NHEJ efficiency positively correlates with $L_{50}$. (f) HR efficiency positively correlates with $L_{50}$

The statistical analysis indicates that young cells are more resistant to IR than old cells $\left(\mathrm{LD}_{50}, \quad P<0.0001 ; \mathrm{LD}_{75}\right.$, $P<0.0001$ ) (Figures $4 \mathrm{c}$ and $\mathrm{d}$ ). We then analyzed the correlations between $\mathrm{LD}_{50}$ and NHEJ efficiency and HR efficiency. We observed a significant negative correlation between $L_{50}$ and both NHEJ and HR efficiency (NHEJ versus $\mathrm{LD}_{50}, P<0.05$; $\mathrm{HR}$ versus $\mathrm{LD}_{50}, P<0.01$ ) (Figures $4 \mathrm{e}$ and f), strongly suggesting that the age-related decline of DNA repair by NHEJ and HR contributes to the rise of radiosensitivity with age.

Declined expression of NHEJ factors is responsible for the age-related impairment of NHEJ. To elucidate the regulatory mechanisms underlying the negative association 
between NHEJ and age, we first compared protein expression levels of major NHEJ factors between the two groups. We found that expression of XRCC4 and DNA Lig4, which form a complex to ligate the broken ends during the NHEJ process, is significantly higher in the young group than the aged group (Figures $5 \mathrm{a}$ and b). However, no significant difference between the two groups was observed for other NHEJ factors (Figure 5a; Supplementary Figure S5). To further confirm that gradual loss of XRCC4 and DNA Lig4 expression with age is responsible for the decline of NHEJ efficiency and fidelity, we knocked down endogenous XRCC4 and DNA Lig4, respectively, in one of the young cell lines (Figure 5c). Surprisingly, we observed no change of NHEJ efficiency with both proteins depleted (Figure $5 \mathrm{~d}$ ), whereas knocking down both genes impairs the NHEJ fidelity (Figure 5e). As alt-NHEJ is also capable of repairing DNA DSBs, we hypothesized that both c-NHEJ and alt-NHEJ are down-regulated with age, therefore leading to the decrease of NHEJ efficiency. To test this hypothesis, we examined the expression of important alt-NHEJ factors including PARP1 and DNA Lig3 in the 28 samples (Figures $5 f$ and g). We found that DNA Lig3 is also down-regulated, demonstrating that the decreased expression of both c-NHEJ and alt-NHEJ factors causes the decline of NHEJ efficiency. We next examined if overexpression of XRCC4 and DNA Lig4 may rescue the decline of NHEJ efficiency and fidelity in an aged cell line. The ORF of XRCC4 and DNA Lig4 were cloned under a CMV promoter and expressed in the aged cell line (Figure $5 \mathrm{~h}$ ). We found that a combinatory overexpression of both XRCC4 and DNA Lig4 promotes both efficiency and fidelity of NHEJ (Figure 5i). Instead, overexpressing only XRCC4 or DNA Lig4 had no significant effect on either efficiency or fidelity, confirming that the age-related decline of both efficiency and fidelity is a result of reduction of both factors in protein expression.

Age-associated impaired recruitment of Rad51 to DNA damage sites contributes to the decline of HR efficiency during aging. To understand the molecular mechanisms of the age-related decline of HR efficiency, we first performed the western blot analysis to examine the expression of essential HR factors including BRCA1, MRE11, Rad50, NBS1, Rad51, and then compared the expression levels of these proteins between the two groups (Figure 6a). We did not observe any significant differences in the expression levels of any HR repair factors (Supplementary Figure S5), suggesting that change in HR efficiency is probably not due to the alteration of protein expression.

Another possible cause of the change in HR efficiency during aging is altered cell cycle distribution as HR occurs only in the S/G2/M stages, when sister chromatids are present. ${ }^{15}$ To rule out this possibility, we compared cell cycle distribution between the 28 cell lines. We observed no significant difference of cell cycle distribution in S/G2/M between two groups (Supplementary Figure S6).

During the process of repairing DNA DSBs by HR, important factors such as Rad51, the major recombinase in this pathway, are recruited to the lesion sites in response to DNA damages. Failed recruitment of essential factors to the damage sites would result in the decline of HR efficiency. We therefore hypothesized that age may potentially impair the recruitment of HR factors. We tested the recruitment of Rad51 to DSB sites at different time points $(0,2,8$ and $24 \mathrm{~h})$ post IR (Figures $6 b-d)$. We found an average of 23 Rad51 foci per nucleus in the young at $2 \mathrm{~h}$ post IR; by contrast older cells exhibited only 16.88 Rad51 foci at the same time point. At $8 \mathrm{~h}$ post IR, the Rad51 foci number was reduced to 9.11 and 6.20 , respectively, whereas at $24 \mathrm{~h}$ post IR, no significant difference was observed between the two groups (Supplementary Figure S7). Taken together, these results strongly suggest that although the HR machinery is still functional in older cells, the impaired recruitment of Rad51 at early time points post IR contributes to the age-associated decline of HR efficiency.

Simultaneous overexpression of both XRCC4 and DNA Lig4 suppresses stress-induced premature cellular senescence. Declines in DNA repair capacities by NHEJ and HR cause the rise of genomic instability, leading to stress-induced premature cellular senescence (SIPS), which contributes to organismal aging. ${ }^{25}$ We therefore hypothesized that promoting DNA DSB repair may suppress the formation of SIPS. As it is easier to restore the declined expression of NHEJ factors, and NHEJ is the dominant pathway in mammals, we examined if overexpression of both XRCC4 and DNA Lig4 could potentially ameliorate the onset of SIPS. We then treated a young and an old cell line with IR, at doses of 10 and $30 \mathrm{~Gy}$, followed by the analysis of $\beta$-gal staining and EdU incorporation. We found that although $10 \mathrm{~Gy}$ IR treatment results in a low percentage of SIPS cells, overexpression of the two NHEJ factors significantly suppresses the formation of SIPS by $23 \%$ in young cells and $30 \%$ in old cells. At the higher dose of IR, we observed a higher fraction of cells underwent SIPS and a similarly robust protective effect of overexpressing two NHEJ factors (24\% in young cells and $16 \%$ old cells) (Figures $7 a$ and $b$ ).

In addition, we performed an EdU assay to examine the division rates of irradiated cells in the presence or absence of XRCC4 and Lig4 overexpression. Consistent with the analysis of $\beta$-gal staining, we found that overexpression of the two NHEJ factors stimulated the proliferation rate by 56 and $45 \%$ at a dosage of 10 and 30 Gy in comparison to that of control cells (Figure 7c).

Finally, we analyzed the expression level of p16, a biomarker for cellular senescence. Surprisingly, western blot analysis of p16 expression level is much more striking. We observed a strong reduction of $\mathrm{p} 16$ expression in XRCC4 and Lig4 overexpressing cells upon X-ray treatment (Figure 7d), bolstering the hypothesis that promoting the NHEJ pathway is a promising way to suppress SIPS, and therefore delaying aging.

\section{Discussion}

Although great attention has been paid to the potential relationship between aging and DNA DSB repair, the major descriptive and mechanistic studies were performed in rodent models. $^{3,4,6,11,17,23}$ Relevant research in humans was mainly focused on age-related change in the recruitment kinetics of essential DNA damage response factors, assayed by immune-staining; ${ }^{26}$ age-related change of genomic instability, 
measured by comet assay; ${ }^{7}$ age-related change of expression profile of important DNA repair factors, analyzed by RNA array and proteomic tools. ${ }^{27,28}$ Although the previous work greatly advanced our understanding of age-associated changes of DNA DSB repair, due to a lack of proper tools for the analysis of NHEJ and HR efficiency and fidelity separately, and the hardship of acquiring a sufficient number of human samples, whether NHEJ efficiency and fidelity, and HR efficiency change with age in humans and the consequences of any such change, and its underlying molecular mechanism are not well understood. Here, we established 50 eyelids fibroblast cell lines derived from donors who are evenly distributed by age. With these cell lines, using our well-characterized reporters for the analysis of NHEJ and HR capacities, for the first time, we conclusively demonstrate that both DNA repair pathways decline with age. The impaired recruitment of Rad51 a

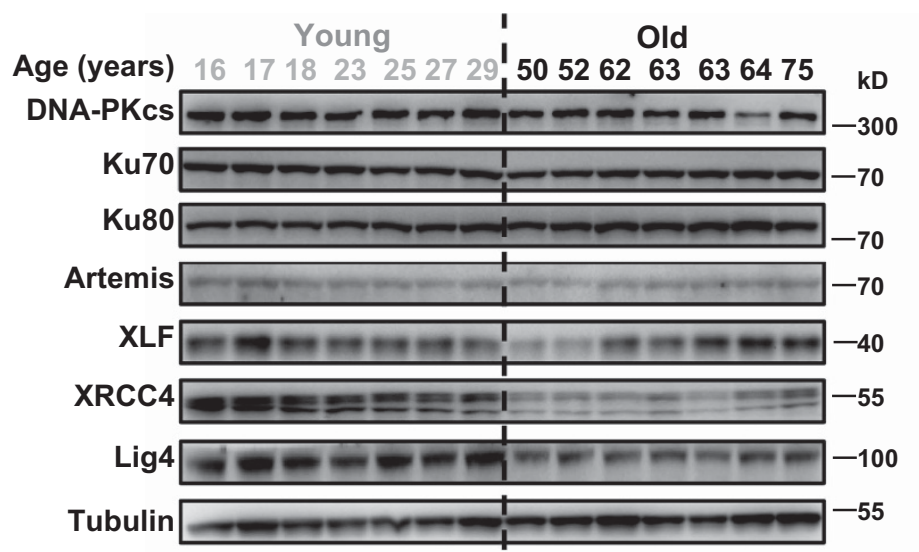

C

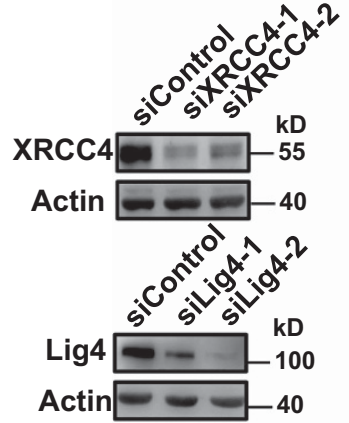

e

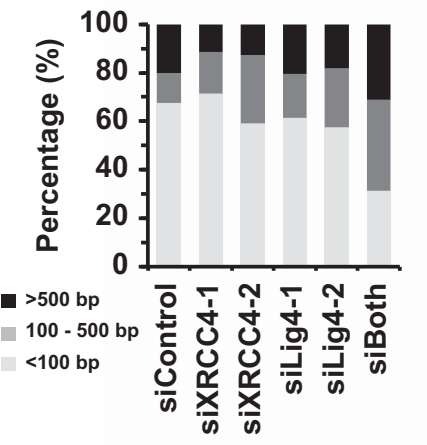

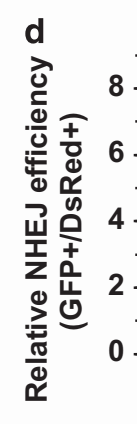

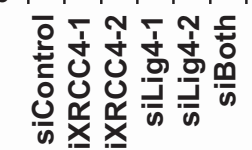

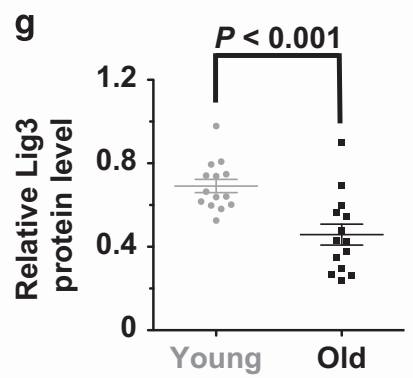
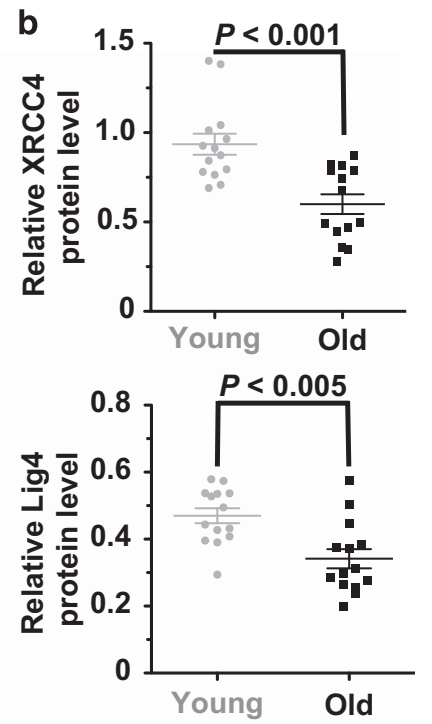

f

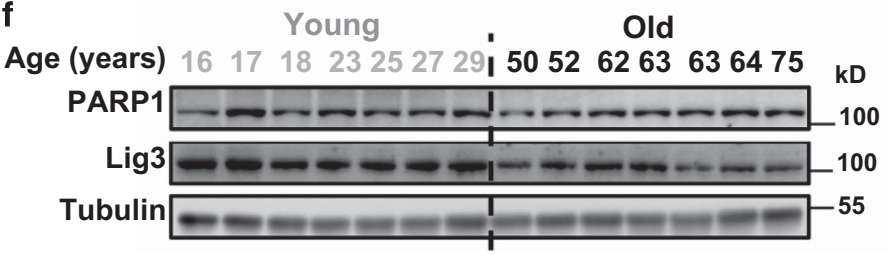

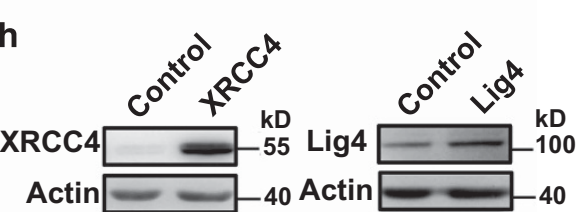
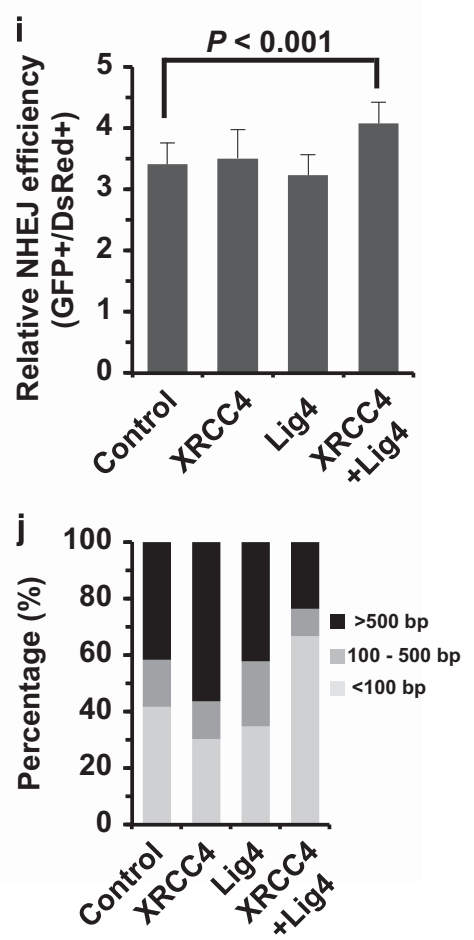
to DNA damage sites during aging hampers the ability of aged cells to choose the precise HR pathway, forcing cells to utilize the error-prone NHEJ pathway. Simultaneously, because of decreased expression of XRCC4, DNA Lig4 and DNA Lig3 during aging, NHEJ becomes more inefficient and inaccurate with age, leaving more damage sites repaired with a loss of more genetic information. The declined DNA DSB repair by both pathways then leads to accumulation of DNA mutations, posing more damages to both NHEJ and HR repair machineries, eventually exacerbating the age-related rise of genomic instability (Figure 8).

Our previous reports indicate that the efficiency of DNA DSB repair by NHEJ and HR declines, and NHEJ becomes more error-prone with replicative cellular senescence. ${ }^{21,29}$ In presenescent cells, HR efficiency declines by 38-fold, whereas NHEJ changes by only two to threefold. Consistent with the above results, our current aging study also shows a sharp decline of HR efficiency during aging, with the biggest difference of an $\sim 30$-fold change, whereas the change of NHEJ with age is relatively mild, albeit statistically significant. However, contradictorily, knocking out major NHEJ factors, such as DNA-PKcs, Ku70, Ku80 or Artemis in mice leads to a phenotype of progeria, ${ }^{4}$ whereas knocking out HR factors usually leads to a phenotype of embryonic lethality, 4,30,31 suggesting that NHEJ is more likely to be involved in aging. Considering an organism's life history is likely critical for reconciling these observations. During embryogenesis cells are rapidly dividing and therefore undergoing replication stress; complete loss of HR, which is a dominant pathway for relieving replication stress, ${ }^{32}$ may cause cells to enter apoptosis by activating P53, leading to embryonic lethality. However, the embryonic lethality could mask the roles of HR in aging. Indeed, partial loss of HR might also lead to agingassociated phenotypes. For instance, BRCA1 heterozygous mice are short lived and have a premature aging phenotype in the ovaries. ${ }^{33,34}$ Intriguingly, once an organism has developed into adulthood, a gradual suppression of the HR pathway with age is needed to counteract the potential tumorigenesis as uncontrolled or overactive single-strand annealing (SSA), which shares almost identical repair machinery with the HR pathway, ${ }^{35}$ may cause loss of large genomic fragments due to the prevalence of repetitive sequences in human genomes.
Our mechanistic study indicates that down-regulated expression of XRCC4 and DNA Lig4, which form a complex to ligate the broken ends, rather than other NHEJ factors such as DNA-PKcs, Ku70 or Ku80, is the major factor contributing to the decline of NHEJ efficiency and fidelity in humans. It is worth noting, however, that XRCC4 or DNA Lig4 deficiency in mice results in embryonic lethality, ${ }^{36}$ whereas mice with other major NHEJ factors knocked out exhibit a phenotype of premature aging. Moreover, in rodents, the expression of $\mathrm{Ku}$ factors usually declines with age. ${ }^{4}$ The reason why we observe such discrepancy probably lies in the differences between species. In comparison to rodents, humans are particularly resistant to tumorigenesis. Early recruitment of DNA-PKcs, Ku heterodimers at DNA DSB sites blocks the SSA and alt-NHEJ repair machineries from accessing the lesions, ${ }^{37,38}$ leaving the ends to be fixed by XRCC4-Lig4 complex. Loss of DNA-PKcs or Ku factors may tip the repair toward extremely mutagenic SSA or alt-NHEJ, ${ }^{37,38}$ increasing the probability of tumorigenesis. Therefore, if the age-related decline of NHEJ is inevitable, it is wiser for humans to evolve a 'lesser of two evils' mechanism by which DNA-PKcs or Ku factors do not change with age, allowing the suppression of tumorigenesis, while XRCC4 and Lig4 expression is altered with age.

Cellular senescence, including replicative senescence and SIPS, is one of major sources causing organismal aging. ${ }^{25}$ As SIPS occurs quite often due to the endogenous and exogenous disturbance, whereas most of adult fibroblasts are in quiescent stages rather than under rapid proliferation, ${ }^{39,40}$ suppressing SIPS by promoting genomic stability may be an intriguing target to delay aging. Indeed, a recent report indicates that ATM, the major sensor of DNA DSBs, suppresses the protein stability of ARF, which may trigger the onset of cellular senescence. ${ }^{41}$ This suggests that there is a functional interplay between genome integrity and cellular senescence. Consistent with the previous findings-although the regulatory mechanisms remain undetermined-our results of suppressing $\mathrm{p} 16^{\mathrm{INK} 4 a}$ by restoring XRCC4 and DNA Lig4 also demonstrate that stabilizing genome integrity by promoting $\mathrm{c}-\mathrm{NHEJ}$ delays the onset of cellular senescence. Although a mouse model with XRCC4 and DNA Lig4 overexpressed is still needed to confirm if targeting the two proteins could extend lifespan or improve healthspan, our data

Figure 5 Age-related reduction of XRCC4, Lig4 and Lig3 expression causes the decline of NHEJ capacity. (a) Western blot analysis of c-NHEJ factors in the two groups of cells. Cells were collected for the whole-cell lysate extraction and western blot analysis at $48 \mathrm{~h}$ post splitting. (b) Statistical comparison of XRCC4 and Lig4 expression between the two groups. Western blot results were further analyzed with ImageJ software (Bethesda, MD, USA) for quantification. (c) XRCC4 and Lig4 were successfully depleted in the 17-year-old cell line using siRNA transfections. Cells were transfected with siRNA twice with two days interval. On day 3 post the second siRNA transfection, cells were collected for protein extraction and western blot analysis. (d) No significant effect on NHEJ efficiency was observed with the two proteins depleted. On day 2 post the second siRNA transfection, $3 \times 10^{5}$ cells were transfected with $0.2 \mu \mathrm{g} \mathrm{I-Scel} \mathrm{linearized} \mathrm{NHEJ} \mathrm{reporter} \mathrm{and} 0.005 \mu \mathrm{g}$ pDsRed2-N1. At $72 \mathrm{~h}$ post transfection, cells were collected for the FACS analysis. Error bars, S.D. (e) Depleting both proteins using siRNA causes a reduction of NHEJ fidelity. Similar to the analysis in (d), cells depleted with the indicated proteins using siRNA were further transfected with $0.2 \mu \mathrm{g} \mathrm{I-Scel} \mathrm{linearized} \mathrm{NHEJ} \mathrm{reporter,} \mathrm{followed} \mathrm{by} \mathrm{DNA} \mathrm{extraction} \mathrm{and} \mathrm{plasmid} \mathrm{rescue.} \mathrm{The} \mathrm{repaired} \mathrm{NHEJ} \mathrm{constructs} \mathrm{were} \mathrm{further}$ sequenced at the junction area. At least 20 clones with deletions were sequenced and quantified. (f) Western blot analysis of alt-NHEJ factors in the two groups of cells. (g) Statistical comparison of Lig3 expression between the two groups. (h) Western blot analysis of XRCC4 and Lig4 overexpression in the 63-year-old cell line. Cells at the amount of $1 \times 10^{6}$ were transfected with $5 \mu \mathrm{g} \mathrm{XRCC4}$ or Lig4 expression vector. At $24 \mathrm{~h}$ post transfection, cells were collected for protein extraction and western blot analysis. (i)

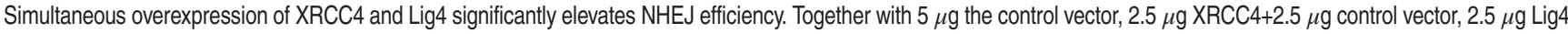

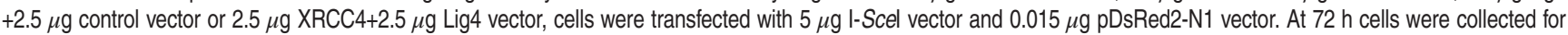
analysis of NHEJ efficiency. All experiments were repeated at least six times. Error bars, S.D. (j) Overexpression of both XRCC4 and Lig4 rescues the decline of NHEJ fidelity in the 63-year-old cell line. Similar to the experiments performed in (i), at $72 \mathrm{~h}$ post co-transfection, cells were collected for DNA extraction and plasmid rescue. For each group, at least 20 clones with deletions were sequenced and further analyzed 

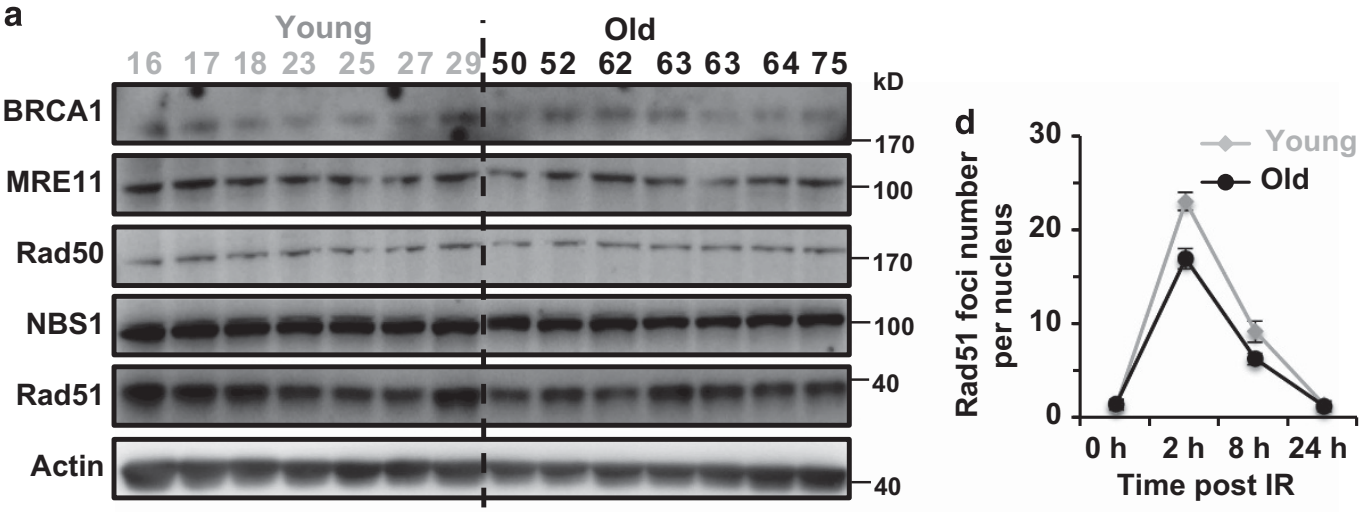

b

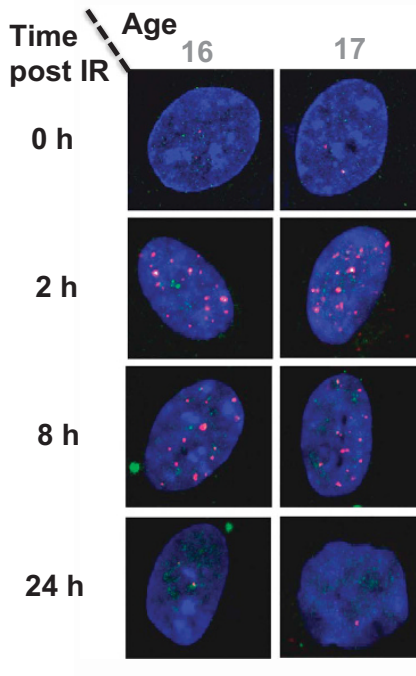

63
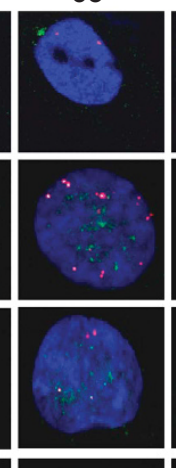

75

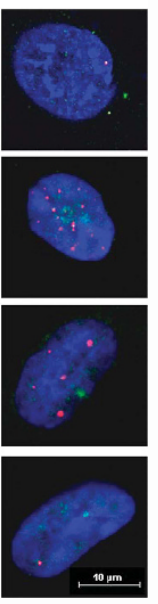

e
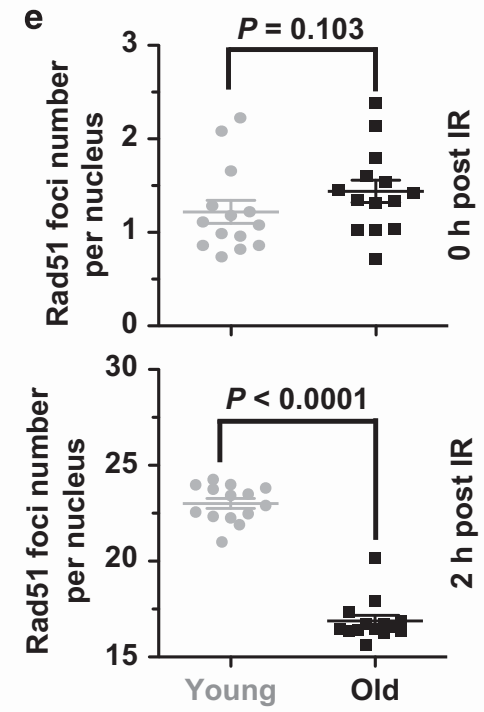

c

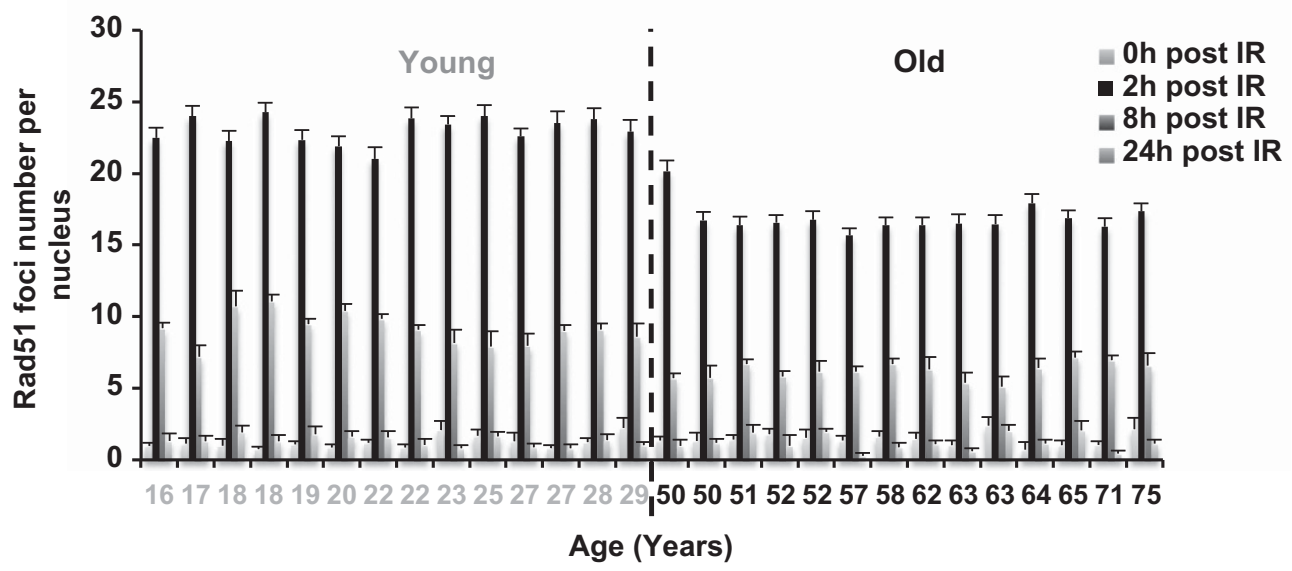

Figure 6 The recruitment of Rad51 to DNA damage sites is impaired in the old group of cells. (a) Western blot analysis of major HR factors in the 28 cell lines. Cells were preseeded and grown for $48 \mathrm{~h}$ before being collected for the protein extraction and the western blot analysis. (b) Representative pictures of Rad51 foci at 0, 8 and $24 \mathrm{~h}$ post IR in two young and two old cell lines. At $48 \mathrm{~h}$ post splitting, cells were treated with X-ray at a dosage of $8 \mathrm{~Gy}$. At different time points post the treatment, cells were fixed and co-stained with Geminin and Rad51 antibodies. Pictures were taken on a confocal microscope. Scale bars, $10 \mu \mathrm{m}$. (c) Quantification of Rad51 foci post IR in the 28 cell lines. At least 50 Gemininpositive cells were counted on the fluorescence microscope. Error bars, S.E.M. (d) The kinetics of Rad51 recruitment to DNA damage sites. Each point represents the average number of Rad51 foci per nucleus for each of the 14 cell lines at different time points post IR. Error bars, S.D. (e) Statistical comparison of Rad51 foci number at $0 \mathrm{~h}$ post IR between the two groups of cells. MWU test was performed to compare the difference. (f) Statistical comparison of Rad51 foci number at $2 \mathrm{~h}$ post IR between the two groups of cells. MWU test was performed to compare the difference 
a

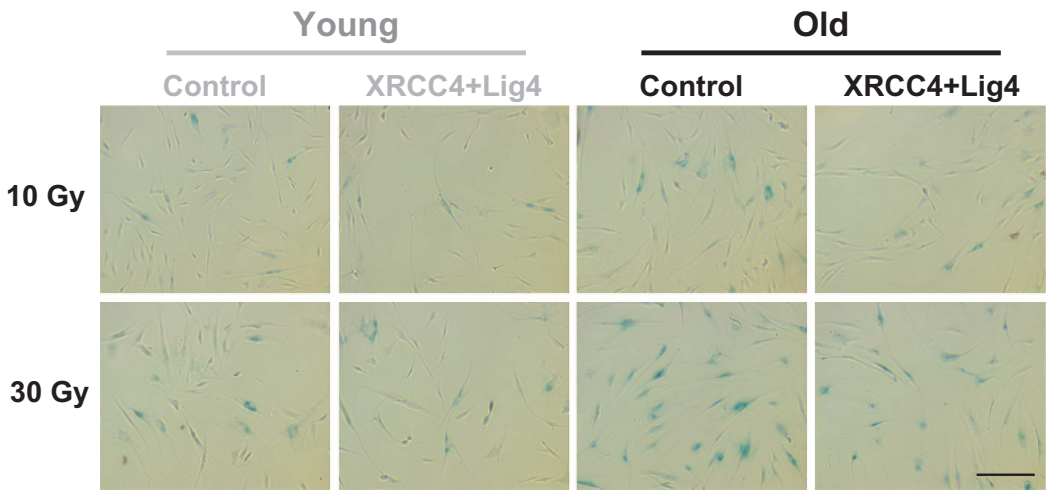

b

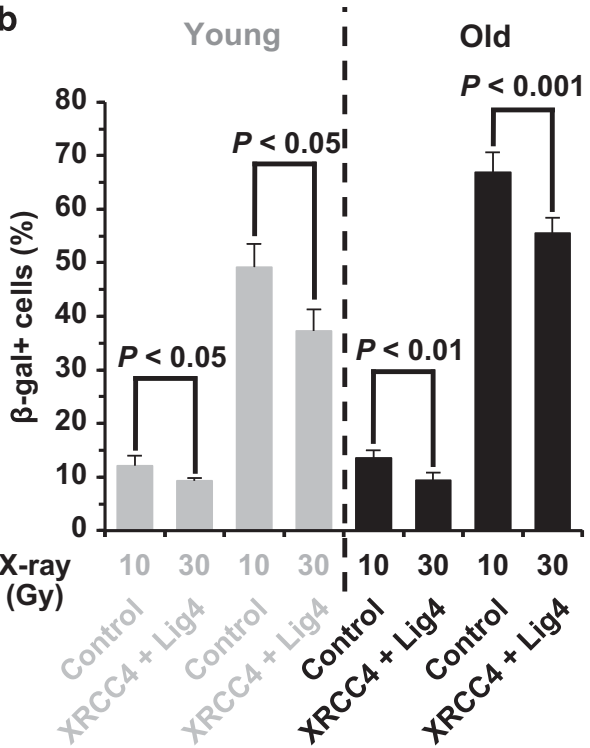

c

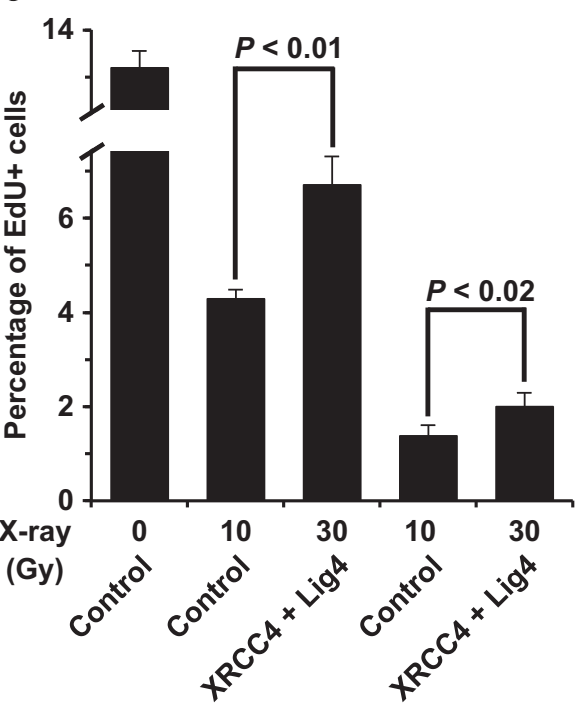

$10 \mathrm{~Gy}$

XRCC4

+Lig4

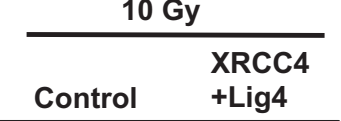

d

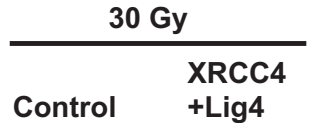

p16

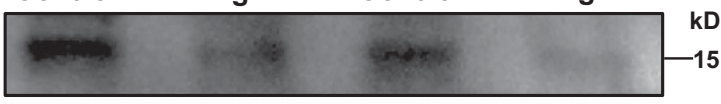

Tubulin

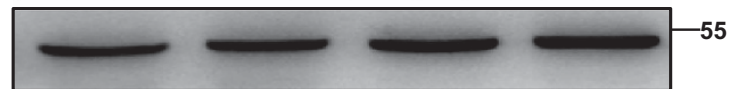

Figure 7 Overexpression of both XRCC4 and Lig4 suppresses the onset of SIPS. (a) Representative pictures of $\beta$-gal staining on 10 or 30 Gy X-ray-treated 17-year-old and 63-year-old fibroblasts. Cells were transfected with control vector or XRCC4+Lig4 expression vectors, and irradiated at indicated dosages, then kept in the incubator for 10 days before being stained. Scale bars, $100 \mu \mathrm{m}$. (b) Comparison of $\beta$-gal+ cell numbers between the control and XRCC4+Lig4 overexpressing cells. The experiments were repeated 3-6 times and a t-test was performed to compare the difference. Error bars, S.D. (c) Analysis of cell division rates on 10 or 30 Gy X-ray-treated 63-year-old fibroblasts using the EdU incorporation assay. (d) Western blot analysis of p16 expression in irradiated cells with control and XRCC4+Lig4 vectors transfected. On day 10 post IR, cells were collected for protein extraction and western blot analysis

at least provides a hint that promoting genomic stability by activating the c-NHEJ pathway holds the potential to delay onset of aging.

In summary, our results not only reveal that an ageassociated decline of DNA DSB repair by two pathways causes the destabilization of genome integrity in humans, but also delineates the regulatory mechanisms of aging-related changes in NHEJ and HR. Our research lays the foundation for developing novel ways of promoting c-NHEJ to stabilize genomic stability, thereby delaying aging and treating agingassociated diseases.

\section{Materials and Methods}

Isolation of human eyelid fibroblast. All human subjects associated with this study participated of their own consent, and in accordance with the Ethic Committees of both Tongji University and Changzheng Hospital. Eyelids were acquired from plastic surgeries and kept in ice-cold PBS; fibroblasts isolation 


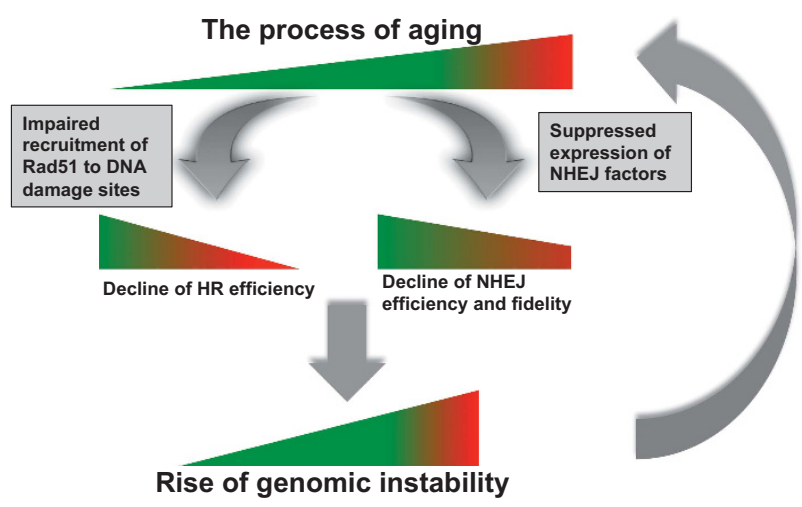

Figure 8 Model for aging, change of DNA DSB repair and the rise of genomic instability. During the process of aging, the accumulation of mutations gradually impairs the recruitment of essential HR factors to DNA damage sites, and the expression of NHEJ factors, causing a decline o DNA DSB repair capacity. The age-related decline of $\mathrm{HR}$ is much shaper than that of NHEJ, possibly acting as a tumor suppressing mechanism. However, it also forces cells to choose the more error-prone NHEJ repair pathway. Decrease of both pathways eventually introduces more DNA mutations to genomes, leading to age-related to genomic instability, accelerating the process of aging

experiments were performed within $24 \mathrm{~h}$. The detailed isolation protocol is as previously reported. ${ }^{18,19}$

Cell culture. All primary fibroblasts were cultured in MEM medium (Hyclone, Logan, UT, USA, Cat. \#SH30234) containing 10\% FBS (Gibco, Carlsbad, CA, USA, Cat. \# 16000) supplemented with $1 \%$ penicillin/streptomycin (Hyclone, cat. \#SV30010) and 1\% NEAA (Hyclone, Cat. \# SH3023801). Cells were maintained in incubators (Thermo Fisher, Waltham, MA, USA, Heracell 240i) with $5 \% \mathrm{CO}_{2}$ and $3 \% \mathrm{O}_{2}$ at $37^{\circ} \mathrm{C}$. All cultured cells were counted on a Millipore Muse machine (Hayward, CA, USA) and the PD number was calculated as previously reported. ${ }^{21,29}$

Plasmids, siRNAs and antibodies. The backbone vectors overexpressing XRCC4 and DNA Lig4 are based on pEGFP-N1. Both XRCC4 and DNA Lig4 open reading frames were amplified from $C D N A$ of $H C A 2$ fibroblast using primers:XRCC45': CTGGTCGACGCCACCATGGAGAGAAAAATAAGCAGA, XRCC4-3': AATGCGG CCGCTTAAATCTCATCAAAGAGGTC and Lig4-5': TTCGTCGACGCCACCATGGC TGCCTCACAAACTTCAC, Lig4-3': GAAGCGGCCGCTTAAATCAAATACTGGTTT TC, followed by replacement of EGFP with the two ORFs using Sall and Nott restriction enzymes. The sequences of siRNAs against XRCC4 and DNA Lig4 are as follows: siXRCC4-1, AUAUGUUGGUGAACUGAGATT; siXRCC4-2, UCUUGGGA CAGAACCUAAATT; siLig4-1, CGACCUUUUAGACUCAAUUTT; siLig4-2, GCUAGA UGGUGAACGUAUGTT. The information of antibodies used in western blot and immunostaining in this study is as follows: $\gamma \mathrm{H} 2 \mathrm{Ax}$ (Abcam, Cambridge, MA, USA, Cat. \# ab20669), DNA-PKcs (Abclonal, College Park, MD, USA, Cat. \# A1419), Ku70 (Abclonal, Cat. \# A0883), Ku80 (Abclonal, Cat. \# A5862), Artemis (Abclonal, Cat. \# A5615), XLF (Abcam, Cat. \# ab189917), XRCC4 (Abclonal, Cat. \# A7539), DNA Lig4 (Abclonal, Cat. \# A1743), PARP1 (Abclonal, Cat. \# A3121), DNA Lig3 (Abclonal, Cat. \# A1887), BRCA1 (Cell signaling, Danvers, MA, USA, Cat. \# 9010S), MRE11 (Abclonal, Cat. \# A2559), Rad50 (Abclonal, Cat. \# A0182), NBS1 (Abclonal, Cat. \# A0783), Rad51 (Abclonal, Cat. \# A6268), Actin (Santa Cruz, Dallas, TX, USA, Cat. \# SC-47778), Tubulin (Abmart, Berkeley Heights, NJ, USA, Cat. \# M20005), p16 (Abcam, Cat. \# ab108349).

Transfections. Cells were split $48 \mathrm{~h}$ before electroporation. For different purposes, a certain number of cells were transfected with indicated amount of NHEJ or HR construct using the electroporation kit (V4XP-2032) on a Lonza 4D machine (Cologne, Germany) (program DT-130).

FACS analysis. For analysis of NHEJ or HR efficiency, cells were collected at $72 \mathrm{~h}$ post transfection, re-suspended in $0.5 \mathrm{ml}$ PBS and kept on ice before being used for analysis on FACSverse (BD Biosciences, San Jose, CA, USA). At least 20000 cells were counted. For analysis of cell cycle distribution, cells were collected at $48 \mathrm{~h}$ post splitting, followed by fixation with $-20^{\circ} \mathrm{C}$ cold $70 \%$ ethanol.
After fixation at $4{ }^{\circ} \mathrm{C}$ for at least $16 \mathrm{~h}$, cells were stained with PI as described. ${ }^{29}$ In this case, at least 10000 cells were analyzed on FACS. All the data was further analyzed using the software of FlowJo (Ashland, OR, USA).

Comet assay. Cells were collected for the neutral comet assay at $48 \mathrm{~h}$ post seeding. The detailed procedure is as described in the manufacturer's instructions (Trevigen, Gaithersburg, MD, USA, Cat. \# 4250-050-K).

Clonogenic assay. On day 2, post splitting cells were treated with increasing doses of $X$-ray at a dose of $0,2,4,6$, and 8 Gy. Irradiated cells were immediately plated after serial dilutions. Cells were cultured in the incubators for 2 weeks before staining with Commassie reagent (methanol : acetic acid : Commassie : $\mathrm{H}_{2} \mathrm{O}=50: 10: 0.25: 40$ ) for $3 \mathrm{~h}$. Afterwards, stained cells were washed with distilled water and colonies with at least 50 cells were counted. Survival rate was calculated as the ratio of the colonies number of the irradiated to that of control cells.

$\boldsymbol{\beta}$-gal staining. Cells were fixed in $2 \%$ formaldehyde, $0.2 \%$ glutaraldehyde in PBS for $5 \mathrm{~min}$ at room temperature, followed by two PBS washes. Then staining solution (1 mg/ml X-gal in dimethylformamide, $40 \mathrm{~mm}$ citric acid/Na phosphate buffer, $5 \mathrm{~mm}$ potassium ferrocyanide, $5 \mathrm{~mm}$ potassium ferricyanide, $150 \mathrm{~mm}$ sodium chloride, $2 \mathrm{~mm}$ magnesium chloride) was added and incubated for $16 \mathrm{~h}$ at $37^{\circ} \mathrm{C}$. Pictures of stained cells were taken on a Zeiss inverted microscope (Goettingen, Germany).

EdU incorporation assay. Cells were seeded at a density of $5 \times 10^{5}$ cell/plate 2 days before transfection. Then $5 \mu \mathrm{g}$ of pcDNA3.1 or $2.5 \mu \mathrm{g}$ of a vector encoding XRCC4 and $2.5 \mu \mathrm{g}$ of a vector encoding DNA Lig4 were transfected to fibroblasts. On day 1 post transfection, cells were irradiated with $\mathrm{X}$-ray at a dosage of $0,5,10$, and 30 Gy. Forty-eight hours later, cells were incubated with $10 \mu \mathrm{M}$ EdU for $2 \mathrm{~h}$, followed by the analysis on FACSverse using a Click-iT EdU Assay Kit (Invitrogen, Waltham, MA, USA, C10634).

\section{Conflict of Interest}

The authors declare no conflict of interest.

Acknowledgements. We thank Dr Michael Van Meter for critically reading the manuscript. This work was supported by grants from National Basic Research Program of China (973 Program, Grant Nos. 2013CB967600, 2015CB964800), the National Science Foundation of China (Grant Nos. 31371396, 31570813, 91519319), Shanghai Pujlang Program (Grant Nos. 13PJ1408300), the 1000 Youth Talents Program, the Fundamental Research Funds for the Central Universities, the Open Project Program of State Key Laboratory of Natural Medicines, China Pharmaceutical University (Grant No. SKLNMKF201404) to ZM, and a grant from National Undergraduate Training Programs for Innovation and Entrepreneurship (201410247081) to YC.

1. Lopez-Otin C, Blasco MA, Partridge L, Serrano M, Kroemer G. The hallmarks of aging. Cell 2013; 153: 1194-1217.

2. Tigges J, Krutmann J, Fritsche E, Haendeler J, Schaal H, Fischer JW et al. The hallmarks of fibroblast ageing. Mech Ageing Dev 2014; 138: 26-44.

3. Dolle ME, Giese H, Hopkins CL, Martus HJ, Hausdorff JM, Vijg J. Rapid accumulation of genome rearrangements in liver but not in brain of old mice. Nat Genet 1997; 17: 431-434.

4. Gorbunova V, Seluanov A, Mao Z, Hine C. Changes in DNA repair during aging. Nucleic Acids Res 2007; 35: 7466-7474.

5. Rube CE, Fricke A, Widmann TA, Furst T, Madry H, Pfreundschuh $\mathrm{M}$ et al. Accumulation of DNA damage in hematopoietic stem and progenitor cells during human aging. PLOS ONE 2011; 6: e17487.

6. Wang C, Jurk D, Maddick M, Nelson G, Martin-Ruiz C, von Zglinicki T. DNA damage response and cellular senescence in tissues of aging mice. Aging Cell 2009; 8: 311-323.

7. Garm C, Moreno-Villanueva M, Burkle A, Petersen I, Bohr VA, Christensen K et al. Age and gender effects on DNA strand break repair in peripheral blood mononuclear cells. Aging Cell 2013; 12: 58-66.

8. Mayer PJ, Lange CS, Bradley MO, Nichols WW. Age-dependent decline in rejoining of X-rayinduced DNA double-strand breaks in normal human lymphocytes. Mutat Res 1989; 219: 95-100.

9. Ren K, Pena de Ortiz S. Non-homologous DNA end joining in the mature rat brain. $\checkmark$ Neurochem 2002; 80: 949-959.

10. Singh NP, Danner DB, Tice RR, Brant L, Schneider EL. DNA damage and repair with age in individual human lymphocytes. Mutat Res 1990; 237: 123-130.

11. Vyjayanti VN, Rao KS. DNA double strand break repair in brain: reduced NHEJ activity in aging rat neurons. Neurosci Lett 2006; 393: 18-22. 
12. White RR, Milholland B, de Bruin A, Curran S, Laberge RM, van Steeg $\mathrm{H}$ et al. Controlled induction of DNA double-strand breaks in the mouse liver induces features of tissue ageing. Nat Commun 2015: 6: 6790

13. Frit $P$, Barboule N, Yuan $Y$, Gomez D, Calsou P. Alternative end-joining pathway(s): bricolage at DNA breaks. DNA Repair 2014; 17: 81-97.

14. Waters CA, Strande NT, Wyatt DW, Pryor JM, Ramsden DA. Nonhomologous end joining: a good solution for bad ends. DNA Repair 2014; 17: 39-51.

15. Mao Z, Bozzella M, Seluanov A, Gorbunova V. DNA repair by nonhomologous end joining and homologous recombination during cell cycle in human cells. Cell Cycle 2008; 7: 2902-2906.

16. Jasin M, Rothstein R. Repair of strand breaks by homologous recombination. Cold Spring Harb Perspect Biol 2013; 5: a012740.

17. Gorbunova V, Seluanov A. Making ends meet in old age: DSB repair and aging. Mech Ageing Dev 2005; 126: 621-628.

18. Seluanov A, Hine C, Bozzella M, Hall A, Sasahara TH, Ribeiro AA et al. Distinct tumor suppressor mechanisms evolve in rodent species that differ in size and lifespan. Aging Cell 2008; 7: 813-823.

19. Xu Z, Zhang L, Zhang W, Meng D, Zhang H, Jiang $Y$ et al. SIRT6 rescues the age related decline in base excision repair in a PARP1-dependent manner. Cell Cycle 2015; 14: 269-276.

20. Mao Z, Hine C, Tian X, Van Meter M, Au M, Vaidya A et al. SIRT6 promotes DNA repair under stress by activating PARP1. Science 2011; 332: 1443-1446.

21. Seluanov A, Mittelman D, Pereira-Smith OM, Wilson JH, Gorbunova V. DNA end joining becomes less efficient and more error-prone during cellular senescence. Proc Natl Acad Sci USA 2004; 101: 7624-7629.

22. Mao Z, Seluanov A, Jiang Y, Gorbunova V. TRF2 is required for repair of nontelomeric DNA double-strand breaks by homologous recombination. Proc Natl Acad Sci USA 2007; 104 13068-13073

23. Vaidya A, Mao Z, Tian X, Spencer B, Seluanov A, Gorbunova V. Knock-in reporter mice demonstrate that DNA repair by non-homologous end joining declines with age. PLoS Genet 2014; 10: e1004511.

24. Cann KL, Dellaire G. Heterochromatin and the DNA damage response: the need to relax. Biochem Cell Biol 2011; 89: 45-60.

25. Campisi J. Senescent cells, tumor suppression, and organismal aging: good citizens, bad neighbors. Cell 2005; 120: 513-522.

26. Sedelnikova OA, Horikawa I, Redon C, Nakamura A, Zimonjic DB, Popescu NC et al. Delayed kinetics of DNA double-strand break processing in normal and pathological aging. Aging Cell 2008; 7: 89-100.

27. Waldera-Lupa DM, Kalfalah F, Florea AM, Sass S, Kruse F, Rieder V et al. Proteome-wide analysis reveals an age-associated cellular phenotype of in situ aged human fibroblasts. Aging 2014; 6: 856-878.
28. Kalfalah F, Seggewiss S, Walter R, Tigges J, Moreno-Villanueva M, Burkle A et al. Structural chromosome abnormalities, increased DNA strand breaks and DNA strand break repair deficiency in dermal fibroblasts from old female human donors. Aging 2015; 7 : 110-122.

29. Mao Z, Tian X, Van Meter M, Ke Z, Gorbunova V, Seluanov A. Sirtuin 6 (SIRT6) rescues the decline of homologous recombination repair during replicative senescence. Proc Natl Acad Sci USA 2012; 109: 11800-11805.

30. Kuznetsov SG, Haines DC, Martin BK, Sharan SK. Loss of Rad51c leads to embryonic lethality and modulation of Trp53-dependent tumorigenesis in mice. Cancer Res 2009; 69: 863-872.

31. Lim DS, Hasty P. A mutation in mouse rad51 results in an early embryonic lethal that is suppressed by a mutation in p53. Mol Cell Biol 1996; 16: 7133-7143.

32. Carr AM, Lambert S. Replication stress-induced genome instability: the dark side of replication maintenance by homologous recombination. J Mol Biol 2013; 425 : 4733-4744.

33. Jeng YM, Cai-Ng S, Li A, Furuta S, Chew H, Chen PL et al. Brca1 heterozygous mice have shortened life span and are prone to ovarian tumorigenesis with haploinsufficiency upon ionizing irradiation. Oncogene 2007; 26: 6160-6166.

34. Titus S, Li F, Stobezki R, Akula K, Unsal E, Jeong K et al. Impairment of BRCA1-related DNA double-strand break repair leads to ovarian aging in mice and humans. Sci Transl Med 2013; 5: 172 ra121.

35. Wyman C, Kanaar R. DNA double-strand break repair: all's well that ends well. Ann Rev Genet 2006; 40: 363-383.

36. Barnes DE, Stamp G, Rosewell I, Denzel A, Lindahl T. Targeted disruption of the gene encoding DNA ligase IV leads to lethality in embryonic mice. Curr Biol 1998; 8 : 1395-1398.

37. Mansour WY, Schumacher S, Rosskopf R, Rhein T, Schmidt-Petersen F, Gatzemeier F et al. Hierarchy of nonhomologous end-joining, single-strand annealing and gene conversion at site-directed DNA double-strand breaks. Nucleic Acids Res 2008; 36: 4088-4098.

38. Fattah F, Lee EH, Weisensel N, Wang Y, Lichter N, Hendrickson EA. Ku regulates the nonhomologous end joining pathway choice of DNA double-strand break repair in human somatic cells. PLOS Genet 2010; 6: e1000855.

39. Cristofalo VJ, Allen RG. Pignolo RJ, Martin BG, Beck JC. Relationship between donor age and the replicative lifespan of human cells in culture: a reevaluation. Proc Natl Acad Sci USA 1998; 95: 10614-10619.

40. Maier AB, le Cessie S, de Koning-Treurniet C, Blom J, Westendorp RG, van Heemst D. Persistence of high-replicative capacity in cultured fibroblasts from nonagenarians. Aging Cell 2007; 6: 27-33.

41. Velimezi G, Liontos M, Vougas K, Roumeliotis T, Bartkova J, Sideridou M et al. Functiona interplay between the DNA-damage-response kinase ATM and ARF tumour suppressor protein in human cancer. Nat Cell Biol 2013; 15: 967-977.

Supplementary Information accompanies this paper on Cell Death and Differentiation website (http://www.nature.com/cdd) 